\title{
Comparison of genital and respiratory carriage of Haemophilus parainfluenzae in men
}

\author{
E. HOUANG, M.PHILIPPOU and Z. AHMET
}

- Department of Microbiology, Chelsea Hospital for Women, Dovehouse Street, London SW3

\begin{abstract}
Summary. In the first study, genital carriage of Haemophilus parainfluenzae was investigated in 103 women and 292 men attending a clinic for genitourinary medicine. In a second study, pairs of urethral and throat swabs were studied in 279 men. The vaginal carriage was $2 \%$, urethral $12.4 \%$ and throat $13.3 \%$. Biotype 2 was found to be a genital type and throat carriage of this biotype was significantly associated with its concomitant urethral carriage. Biotypes 1 and 3 were mainly found in the throat. Biotype 2 was significantly more likely to be resistant to ampicillin, tetracycline and sulphonamide but biotype 1 was significantly more likely to be resistant to trimethoprim.
\end{abstract}

\section{Introduction}

Haemophilus influenzae is a recognised respiratory pathogen and is also occasionally responsible for genitourinary infection. Albritton et al. (1982) reported that $H$. influenzae strains isolated from the genitourinary tract generally differed in biotype from respiratory isolates. Antibiotic-susceptible genitourinary strains contained plasmids more frequently than antibiotic-susceptible respiratory strains. $H$. parainfluenzae is generally regarded as a commensal in the respiratory tract and may cause sporadic local or systemic infections. Detailed studies of the distribution of biotypes in different body sites and their patterns of antibiotic sensitivity have not so far been carried out. We report the results of a study of respiratory and genital isolates of $H$. parainfluenzae in patients attending a clinic for genitourinary medicine.

\section{Patients and methods}

\section{Study 1}

The first study was performed from October 1983 to January 1984. A vaginal or urethral swab was taken from randomly chosen female (103) and male (292) patients attending the clinic for genitourinary medicine, St Stephen's Hospital, London. Swabs were inoculated immediately after collection on to $7 \% \mathrm{v} / \mathrm{v}$ heated horseblood Columbia Agar (Oxoid) in halved plates, one half of which contained vancomycin $5 \mathrm{mg} / \mathrm{L}$. The plates were incubated immediately for 2 days at $35^{\circ} \mathrm{C}$ in an atmosphere containing $\mathrm{CO}_{2} 7 \%$. Small gram-negative pleomorphic coccobacilli from a single colony were

Received 1 Feb. 1988; accepted with modifications 6 July 1988. examined for X-and V-factor dependency on Nutrient Agar (Oxoid). V-dependent isolates were then tested for the ability to metabolise $\alpha$-aminolaevulinic acid. $H$. parainfluenzae strains thus identified were biotyped by the scheme of Kilian (1976).

\section{Study 2}

The second study was performed from October 1984 to March 1985. A pair of urethral and throat swabs was taken from each of 279 randomly chosen male clinic attenders. The paired swabs were used to inoculate each half of a heated blood-agar plate containing vancomycin $5 \mathrm{mg} / \mathrm{L}$. The plates were incubated as described above. For each specimen, small gram-negative pleomorphic coccobacilli from three separate colonies were examined further. MicroScan HNID panels (American MicroScan, New Jersey) were used for the identification and biotyping of V-dependent isolates.

\section{Antimicrobial susceptibility tests}

Susceptibility to ampicillin, sulphonamide, chloramphenicol, tetracycline and trimethoprim was tested by disk diffusion on lysed blood DST (Oxoid) plates containing NAD $10 \mathrm{mg} / \mathrm{L}$ as described by PhilpottHoward and Williams (1982). The minimum inhibitory concentrations (MICs) of ampicillin, sulphonamide, chloramphenicol, tetracycline and trimethoprim were measured for those strains in the second study which showed a reduced zone diameter $(<20 \mathrm{~mm})$ with one or more of the antibiotics tested (Philpott-Howard and Williams, 1982). For isolates obtained in the first study, MICs of ampicillin only were measured. $\beta$-Lactamase production was tested with a chromogenic cephalosporin (O'Callaghan et al., 1972). Chloramphenicol-resistant isolates were screened for the production of acetyltransferase by the method described by Manten et al. 
(1976). Isolates of the same biotype from the same site but with a more than fourfold difference in the MICs of any antibiotic tested were regarded as distinct from each other.

\section{Results}

In the first study with vaginal and urethral swabs from 103 female and 292 male patients, $H$. parainfluenzae and $H$. influenzae were each isolated from two vaginal swabs $(2 \%)$. In the second study with pairs of urethral and throat swabs from 279 male patients, $H$. parainfluenzae was isolated from both the throat and the urethra of 17 men. The numbers and biotypes of $H$. parainfluenzae obtained from men in both studies are shown in table $\mathrm{I}$.

Throat swabs from five patients and urethral swabs from three patients in the second study yielded more than one biotype of $H$. parainfluenzae. Over the periods of the two studies, a urethral swab was obtained on more than one occasion from 50 men and a throat swab from 11 . The 17 urethral isolates were obtained from 15 men; only two men had positive cultures on more than one occasion, 2 and 12 months between clinic visits respectively. Both had biotype 2 . Of the 22 throat swabs, eight from seven men yielded $H$. parainfluenzae. Only one man had positive cultures on more than one occasion, with colonies of biotype 1 isolated from the first swab and of biotypes 1 and 3 from the second swab 2 months later.

Table II gives the pattern of antibiotic resistance of isolates obtained from the paired urethral and throat specimens. Lancaster and Irwin's method for partitioning $\chi^{2}$ showed that biotype 2 was significantly more likely to be resistant to ampicillin $(\mathrm{p}<0.005)$, tetracycline $(\mathrm{p}<0.01)$ and sulphonamide $(\mathrm{p}<0.01)$ than biotypes 1 and 3 . Biotype 1 was significantly more likely to be resistant to trimethoprim than biotypes 2 and $3(\mathrm{p}<0.001)$. The

Table I. The isolation and distribution of biotypes of $H$.parainfluenzae from the two studies

\begin{tabular}{|c|c|c|c|c|c|c|c|c|c|}
\hline \multirow[b]{2}{*}{ Study no. } & \multirow[b]{2}{*}{ Site } & \multicolumn{2}{|c|}{ Number of patients } & \multicolumn{6}{|c|}{$\begin{array}{c}\text { Number of } H \text {. parainfluenzae isolates of bio- } \\
\text { type }\end{array}$} \\
\hline & & Total & $\begin{array}{c}H . \text { parainfluenzae } \\
\text { positive }\end{array}$ & Total & 1 & 2 & 3 & 4 & NT \\
\hline 1 & $\mathrm{U}$ & 292 & 37 & 37 & 2 & 32 & 3 & 0 & 0 \\
\hline 2 & $\mathrm{U}$ & 279 & 34 & $\begin{array}{c}51 \\
*(30)\end{array}$ & $\begin{array}{c}0 \\
(0)\end{array}$ & $\begin{array}{c}46 \\
(29)\end{array}$ & $\begin{array}{c}3 \\
(1)\end{array}$ & 1 & 1 \\
\hline 2 & $\mathrm{~T}$ & 279 & 37 & $\begin{array}{c}50 \\
(26)\end{array}$ & $\begin{array}{l}14 \\
(7)\end{array}$ & $\begin{array}{c}12 \\
(11)\end{array}$ & $\begin{array}{l}17 \\
(5)\end{array}$ & $\begin{array}{c}7 \\
(3)\end{array}$ & 0 \\
\hline
\end{tabular}

$\mathrm{U}=$ male urethra; $\mathrm{T}=$ male throat.

*( $)=$ Number of isolates from the 17 patients who had positive cultures from both sites.

Table II. Antibiotic susceptibility of $H$. parainfluenzae isolates obtained from the second study

\begin{tabular}{|c|c|c|c|c|c|c|c|c|c|c|c|}
\hline \multirow[b]{3}{*}{ Biotype } & \multirow{3}{*}{$\begin{array}{l}\text { Number of } \\
\text { isolates }\end{array}$} & \multicolumn{10}{|c|}{ Number of isolates with MIC $(\mathrm{mg} / \mathrm{L})$ of } \\
\hline & & \multicolumn{2}{|c|}{ ampicillin } & \multicolumn{2}{|c|}{ chloramphenicol } & \multicolumn{2}{|c|}{ trimethoprim } & \multicolumn{2}{|c|}{ tetracycline } & \multicolumn{2}{|c|}{ sulphonamide } \\
\hline & & $<4$ & $\geqslant 4$ & $<4$ & $\geqslant 4$ & $<4$ & $\geqslant 4$ & $<4$ & $\geqslant 4$ & $<32$ & $\geqslant 32$ \\
\hline 1 & $14(5)^{*}$ & 14 & 0 & 12 & 2 & 10 & $\begin{array}{c}4 \\
(p<0.001) \dagger\end{array}$ & 10 & 4 & 14 & 0 \\
\hline 2 & $58(14)$ & 46 & $\begin{array}{c}12 \\
(p<0.005)\end{array}$ & 49 & 9 & 56 & 2 & 29 & $\begin{array}{c}29 \\
(p<0.01)\end{array}$ & 47 & $\begin{array}{c}11 \\
(p<0.01)\end{array}$ \\
\hline 3 & $20(17)$ & 20 & 0 & 20 & 0 & 20 & 0 & 17 & 3 & 20 & 0 \\
\hline 4 & $8(6)$ & 8 & 0 & 8 & 0 & 8 & 0 & 6 & 2 & 8 & 0 \\
\hline
\end{tabular}

$*()=$ number of isolates with a zone of inhibition $\geq 20 \mathrm{~mm}$ with all antibiotics tested; they are included in the figures under the sensitive range of MICs.

$\dagger p$ value calculated according to Lancaster and Irwin's method for partitioning $\chi^{2}$. Everipp (1977). 
number of isolates of biot; pe 4 was too small to be included in the analysis.

$\beta$-Lactamase was produced by two isolates from the first and three from the second study. All five strains were of biotype 2 and had an MIC of ampicillin $\geqslant 32 \mathrm{mg} / \mathrm{L}$. They were also resistant to chloramphenicol and tetracycline but sensitive to trimethoprim and, with one exception, also to sulphonamide. The six isolates, all of biotype 2, with MICs of chloramphenicol $\geqslant 16 \mathrm{mg} / \mathrm{L}$, were shown to produce chloramphenicol acetyl-transferase.

Five multiresistant isolates were examined by the Plasmid Analysis Unit, Division of Hospital Infection, Central Public Health Laboratory, Colindale, London. Two isolates from the same patient contained no detectable plasmid. Another two isolates from different patients had identical plasmid profiles, containing at least three plasmids of $9.7,3.6$ and $1.5 \mathrm{~kb}$. The fifth isolate also contained the $3 \cdot 6-\mathrm{kb}$ plasmid and possibly a $7 \cdot 1-\mathrm{kb}$ plasmid. None of the plasmids detected were capable of selftransfer.

\section{Discussion}

A study on the respiratory and genital carriage of $H$. parainfluenzae has not so far been reported. We studied randomly chosen patients in the sexually active group who attended the clinic for genitourinary medicine. Because of logistic difficulties, information about the presence or absence of genital symptoms or signs, microbiologically confirmed genital infection, and antibiotic therapy was not obtained for correlation in our survey.

In both studies, heated-blood-agar plates containing vancomycin $5 \mathrm{mg} / \mathrm{L}$ were used for the isolation of $H$. parainfluenzae. Although this medium allowed easy recognition of Haemophilus spp. in male urethral swabs, the presence of vancomycin-resistant commensals made such recognition difficult in swabs from other body sites. This may explain the apparently low vaginal carriage rate of $H$. parainfluenzae and $H$. influenzae $(4 \%$ in our study and $0-2 \%$ in other reports-Albritton et al., 1982; Messing et al., 1983; Lamont et al., 1986). Nevertheless, using heated-blood agar containing vancomycin, bacitracin, clindamycin and amphotericin B, Sturm (1986a) found $8 \%$ of vaginal secretions to contain one or other species of Haemophilus. These two Haemophilus spp. are probably not, therefore, commonly present in the vagina $(<10 \%)$. Of the men we studied, $13 \%$ carried $H$. parainfluenzae in their throats. Chapin and Doern (1983) demonstrated much enhanced isolation of $H$. influenzae from paediatric pharyngeal swabs by including vancomycin, clindamycin and bacitracin in the heated-blood-agar plates. Our results may, therefore, represent an underestimate of the carriage rate of $H$. parainfluenzae in the throat.

In the second study, three Haemophilus colonies randomly chosen from each positive plate were tested for susceptibility to a range of antibiotics, and biotyped. We found that $9 \%$ of men carried multiple biotypes in the urethra and $14 \%$ in the throat. Examination of a greater number of colonies plus the use of a more selective medium for respiratory isolation may yield a greater proportion of men carrying multiple biotypes. Only two of the 16 men were shown to be urethral carriers on more than one occasion. Whether this reflects transient or intermittent urethral carriage is not certain, since the possible effect of antibiotic therapy could not be excluded.

Several authors have reported the predominance of biotype 2 in male genital isolates (Messing et al., 1983; Sturm, 1986a). The results of our study show conclusively that biotype 2 is a genital type. Over $88 \%$ ( 78 of 88 ) of urethral isolates belonged to this type and other biotypes were found only infrequently at this site. Of the 37 men with respiratory carriage of $H$. parainfluenzae, biotype 2 was isolated from nine, and eight of them also had concomitant urethral carriage of type 2 . The occurrence of this type in the throat is therefore significantly associated with its urethral carriage $(p<0 \cdot 001)$. This finding would suggest its orogenital transmission and its ability to survive in the throat; in contrast, the male urethra appears not to offer a favourable environment for other biotypes.

Our results showed that biotypes 1 and 3 are mainly respiratory types. Comparison of our results with those reported so far is difficult because of the difference in the types of specimens and patients studied. Sturm (1986a), who examined sputum samples sent to a clinical laboratory, and Watson et al. (1985), who compared patients with cystic fibrosis or other respiratory infection, both found the commonest biotype in the sputum was 2 (37$43 \%$ ). Oberhofer and Back (1979), on the other hand, found biotype 1 to account for $46 \%, 2$ for $26 \%$ and 3 for $20 \%$ of isolates from sputum samples they examined.

In our study, biotype 2 was significantly more likely to be resistant to ampicillin, tetracycline and sulphonamide than biotypes 1 and 3 , whereas biotype 1 was significantly more likely to be resistant to trimethoprim than biotypes 2 and 3 . Three of the five multiresistant isolates were found to contain plasmids, none of which were capable of self- 
transfer. We did not investigate the possible presence of larger, transmissible plasmids integrated in the host chromosome. Brunton et al. (1986) presented evidence that the small, nonconjugative, $\beta$-lactamase-specifying plasmids of Neisseria gonorrhoeae and Haemophilus spp. are highly related and that phenotypically cryptic plasmids found in several epidemiologically distinct isolates of $H$. parainfluenzae are also related to the $\beta$ lactamase plasmids but do not carry transposon $\mathrm{A}(\operatorname{Tn} A)$ sequences. More recently, Martel et al. (1987) studied three isolates of biotype 2 from the urogenital tract and found them to contain a $\beta$ lactamase-coding $4 \cdot 6-\mathrm{kb}$ plasmid which was identical to the 4.6-kb "African-type" plasmid found in $N$. gonorrhoeae. These strains of $H$. parainfluenzae could, therefore, serve as a reservoir of antibioticresistance plasmids which might then be acquired by $N$. gonorrhoeae or $H$. influenzae present in the urogenital or respiratory tracts.

$H$. parainfluenzae is generally regarded as a commensal of the respiratory tract and has only occasionally been implicated in local or systemic infections, such as pharyngitis, epiglottitis, otitis media, conjunctivitis, dental abscesses, pneumonia, prostatitis, septicaemia, endocarditis, septic arthritis, meningitis, brain abscess, liver abscess and osteomyelitis (Chow et al., 1974; Chunn et al., 1977; Hand, 1979; Chattopadhyay et al., 1983;

\section{REFERENCES}

Albritton W L, Brunton J L, Meier M, Bowman M N, Slancey L A 1982 Haemophilus influenzae: comparison of respiratory tract isolates with genitourinary tract isolates. Journal of Clinical Microbiology 16: 826-831.

Brunton J L, Clare D, Meier M A 1986 Molecular epidemiology of antibiotic resistance plasmids of Haemophilus species and Neisseria gonorrhoeae. Reviews of Infectious Diseases 8 : 713-724.

Chapin K C, Doern G V 1983 Selective media for recovery of Haemophilus influenzae from specimens contaminated with upper respiratory tract microbial flora. Journal of Clinical Microbiology 17: 1163-1165.

Chattopadhyay B, Silverstone P H, Winwood R S 1983 Liver abscess caused by Haemophilus parainfluenzae. Postgraduate Medical Journal 59: 788-789.

Chow A W, Bushkell L L, Yoshikawa T T, Guze L B 1974 Haemophilus parainfluenzae epiglottitis with meningitis and bacteremia in an adult. American Journal of the Medical Sciences 267: 365-368.

Chunn C J, Jones S R, McCutchan J A, Young E J, Gilbert D N 1977 Haemophilus parainfluenzae infective endocarditis. Medicine (Baltimore) 56: 99-113.

Clairmont .G J, Zon L I, Groopman J E 1987 Haemophilus parainfluenzae prostatitis in a homosexual man with chronic lymphadenopathy syndrome and HTLV-111 infection. American Journal of Medicine 82 : 175-178.
Clairmont et al., 1987; Olk et al., 1987). Rhind et al. (1985) suggested that $H$. parainfluenzae is associated with the same clinical spectrum of illness as non-capsulate $H$. influenzae in respiratory infections. A role for $H$. parainfluenzae in non-gonococcal urethritis has been suggested by Fuzi (1980) and Sturm (1986b) but remains to be confirmed by prospective, controlled studies. Like other commensals, $H$. parainfluenzae may be pathogenic in patients who are immunosuppressed, as illustrated by the report (Clairmont et al., 1987) of $H$. parainfluenzae prostatitis in a homosexual man with chronic lymphadenopathy syndrome and HIV infection.

In conclusion, we have shown that $H$. parainfluenzae is carried much less frequently in the vagina than in the male urethra. Biotype 2 is a genital type and is more likely to be resistant to antibiotics than biotypes 1 and 3 which are found mainly in the throat.

We are indebted to Dr A. G. Lawrence, Director of the John Hunter Clinic, St Stephen's Hospital, London, for permission to study his patients and also to all the nursing staff for their invaluable help. We are grateful to Miss $\mathbf{K}$. Ellis and her colleagues in the Plasmid Analysis Unit, Central Public Health Laboratory, Colindale for the plasmid work. We are grateful for the free supply of HNID panels from American MicroScan, New Jersey, USA.
Everipp 1977 Analysis of contingency tables. Chapman and Hall, London, p 41.

Fuzi M 1980 Haemophili in sexually transmitted diseases. Lancet 2: 476.

Hand W L 1979 Haemophilus species In: Mandel G L et al. (eds) Principles and practice of infectious diseases. John Wiley and Sons, New York, pp 1768-1772.

Kilian M 1976 A taxonomic study of the genus Haemophilus, with the proposal of a new species. Journal of General Microbiology 93: 9-62.

Lamont R F, Taylor-Robinson D, Newman M, Wigglesworth J, Elder M G 1986 Spontaneous early preterm labour associated with abnormal genital bacterial colonization. British Journal of Obstetrics and Gynaecology 93: 804-810.

Manten A, van Klingeren B, Dessens-Kroon M 1976 Chloramphenicol resistance in Haemophilus influenzae. Lancet 1: 702.

Martel A Y, Gosselin P, Ouellette M, Roy P H, Bergerson M G 1987 Isolation and molecular characterization of $\beta$-lactamase-producing Haemophilus parainfluenzae from the genital tract. Antimicrobial Agents and Chemotherapy 31: 966968.

Messing M, Sottnek F O, Biddle J W, Schlater L K, Kramer M A, Kraus S J 1983 Isolation of Haemophilus species from the genital tract. Sexually Transmitted Diseases 10: 56-61.

Oberhofer T R, Back A E 1979 Biotypes of Haemophilus encountered in clinical laboratories. Journal of Clinical Microbiology 10: 168-174. 
O'Callaghan C H, Morris A, Kirby S M 1972 Novel method for detection of $\beta$-lactamases by using a chromogenic cephalosporin substrate. Antimicrobial Agents and Chemotherapy 1 : 283-288.

Olk D G, Hamill R J, Proctor R A 1987 Haemophilus parainfluenzae vertebral osteomyelitis. American Journal of the Medical Sciences 294: 114-116.

Philpott-Howard J, Williams J D 1982 Increase in antibiotic resistance in Haemophilus influenzae in the United Kingdom since 1977: report of study group. British Medical Journal 284: 1597-1599.

Rhind G B, Gould G A, Ahmad F, Croughan M J, Calder M A 1985 Haemophilus parainfluenzae and $H$. influenzae respira- tory infection: comparison of clinical features. British Medical Journal 291 : 707-708.

Sturm A W 1986 a Isolation of Haemophilus influenzae and Haemophilus parainfluenzae from genital-tract specimens with a selective culture medium. Journal of Medical Microbiology 21 : 349-352.

Sturm A W 1986b Haemophilus influenzae and Haemophilus parainfluenzae in nongonococcal urethritis. Journal of Infectious Diseases 153: 165-167.

Watson K C, Kerr E J C, Hinks C A 1985 Distribution of biotypes of Haemophilus influenzae and $H$. parainfluenzae in patients with cystic fibrosis. Journal of Clinical Pathology 38: $750-753$. 